\title{
Optical bistability in diode-laser amplifiers and injection-locked laser diodes
}

\author{
Rongqing Hui, Sergio Benedetto, and Ivo Montrosset
}

Dipartimento di Elettronica, Politecnico di Torino, c.so Duca degli Abruzzi 24, 10129 Torino, Italy

Received August 28, 1992

\begin{abstract}
Static and dynamic properties of dispersive optical bistability $(O B)$ in semiconductor lasers biased from below to above threshold have been investigated theoretically. The OB result is found to be varied continuously from below to above threshold with its maximum value located just above threshold; this result has been verified experimentally. A much faster switch-off of $\mathrm{OB}$ can be expected when the laser operates in the injection-locked condition.
\end{abstract}

Optical bistability (OB) in semiconductor lasers and in resonant-type laser amplifiers has attracted much attention recently because of its potential application in optical computing and optical communication networks. When a semiconductor laser, biased just below threshold, is operated as an optical amplifier, dispersive $\mathrm{OB}$ has been demonstrated. ${ }^{1-4}$ On the other hand, OB has also been found recently in optically injection-locked semiconductor lasers. ${ }^{5,6}$ These phenomena have been theoretically explained either when a laser amplifier operates below threshold ${ }^{3,4}$ or when an injection-locked laser works above threshold. ${ }^{5,7}$ Quite different physical images have hitherto been considered for these two cases. Since there is no discontinuity at the threshold of a semiconductor laser, the $\mathrm{OB}$ should be continuous from below to above threshold. However, a unified treatment has not yet been found. In this Letter we present our experimental measurement on the $\mathrm{OB}$ properties of a distributed-feedback semiconductor laser biased from below to above threshold.

The experimental setup is described as follows. Two identical distributed-feedback laser diodes with an emission wavelength of $1554 \mathrm{~nm}$ are used. The first laser (master), biased at three times its threshold, is used to generate the signal light, and its output is injected into the second laser (slave) that works as an OB element; both lasers have one facet antireflection coated and the other facet cleaved. Each laser used is isolated from external reflection with a double-section Faraday optical isolator that provides more than $70 \mathrm{~dB}$ of isolation. A monochromator and a scanning Fabry-Perot interferometer are used for rough and fine measurement of the optical spectrum. Frequency matching and detuning between the two lasers is accomplished by adjusting the heat-sink temperature of the master laser. The injected optical power into the slave laser can be evaluated as follows: first, measure the linewidth-enhancement factor $\alpha$ of the slave laser by using the injectionlocking method ${ }^{8}$; with this $\alpha$ value known, the injected optical power can then be obtained through the measured injection-locking bandwidth when the slave laser is biased above threshold. ${ }^{9}$ At each bias level of the slave laser and with a definite optical power injected from the master, a bistable output from the slave laser can be obtained versus the signal frequency detuning. As an example, the squares in Fig. 1 give the measured bistable loop width versus the relative bias level of the slave laser from below to above threshold while the injected optical power is kept at approximately $-23 \mathrm{dBm}$. Below threshold, the $\mathrm{OB}$ loop increases its width with the increase of the bias level, while at above threshold, the loop width decreases with the bias level. The maximum OB loop width is obtained, in our case, at $\sim 1.03$ times the free-running laser threshold, and this value is, in general, dependent on the amount of injected optical power.

In order to simulate the $O B$ operation, a unified treatment is obviously necessary, which allows one to consider the laser biased from below to above threshold. Our theoretical analysis is based on the rate-equation model of Lang $^{7}$ :

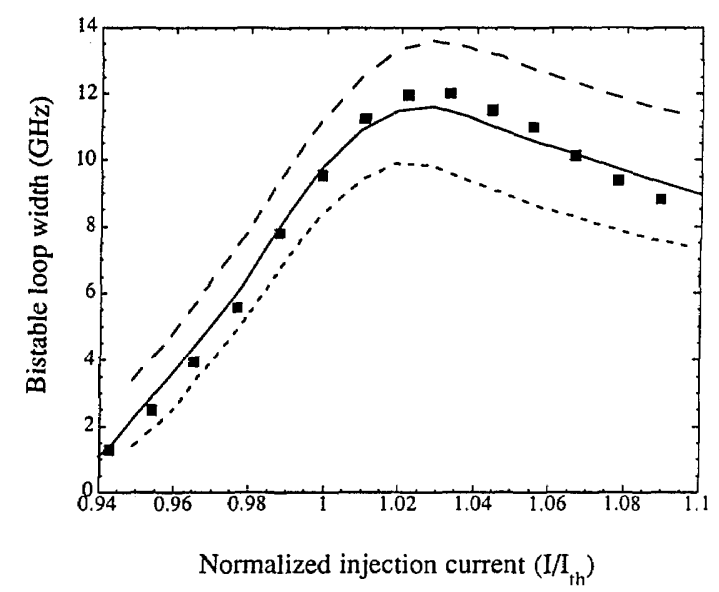

Fig. 1. Measured bistable loop width versus the normalized injection current with the optical injection level at approximately $-23 \mathrm{dBm}$ (squares) together with the calculated results with an injected optical power $P_{\text {in }}$ of $-25 \mathrm{dBm}$ (short-dashed curve), $-23 \mathrm{dBm}$ (solid curve), and $-21 \mathrm{dBm}$ (long-dashed curve). $I_{\text {th }}$ is the free-runing laser threshold. 


$$
\begin{aligned}
\frac{\mathrm{d} E}{\mathrm{~d} t} & =\frac{1}{2}\left[\Gamma G(N, P)-1 / \tau_{p}\right] E+i \Delta \Omega E+\frac{1}{\tau_{i}} E_{\text {ext }}, \\
\frac{\mathrm{d} P_{s}}{\mathrm{~d} t} & =\left[\Gamma G(N, P)-1 / \tau_{p}\right] P_{s}+R_{\mathrm{sp}}, \\
\frac{\mathrm{d} N}{\mathrm{~d} t} & =I / q v-R(N)-G(N, P) P,
\end{aligned}
$$

where $P=P_{s}+|E|^{2}$ is the total photon number in the slave laser's active cavity, $E$ is the normalized electric field generated in response to the externally injected optical source $E_{\text {ext }}, P_{s}$ is the photon number generated in response to the spontaneous emission in Eq. (2), and $\Delta \Omega=\Omega-\omega$ is the relative detuning between the master and the slave lasers' cavity resonance frequency. $G(N, P)=G_{N}\left(N-N_{0}\right)-G_{I} P$ is the material gain, with $G_{N}$ the differential gain and $G_{I}$ the nonlinear gain, $N$ is the carrier number, $N_{0}$ is the carrier number at the transparency. $\tau_{p}$ is the photon lifetime, $\tau_{i}$ is the cavity round-trip time, $\Gamma$ is the confinement factor, $I$ is the electric current, $q$ is the electron charge, and $v$ is the active cavity volume. The carrier dependence of the refractive index is represented by the well-known linewidth-enhancement factor $\alpha=-2(\delta \omega / \delta N) / G_{N}$. Unlike the previous analysis that typically assumed a constant carrier lifetime, carrier-dependent recombination is assumed in our treatment, which appears to be important in fitting the theoretical results with the experimental ones. $R(N)=A N+B N^{2}+$ $C N^{3}$ represents the carrier recombination effect with $A, B$, and $C$ the nonradiative, radiative, and Auger recombination coefficients, respectively. The spontaneous emission rate in Eq. (2) can therefore be assumed to be $R_{\mathrm{sp}}=\beta_{\mathrm{sp}} B N^{2}$, where $\beta_{\mathrm{sp}}$ is the spontaneous emission coefficient, which represents the fraction of spontaneous emission coupled into the lasing mode.

To solve the rate equations (1)-(3), small-signal analysis was usually employed around the condition $\Gamma g\left(N_{\text {th }}\right)=1 / \tau_{p}, 5,7$ where $\Gamma g\left(N_{\text {th }}\right)$ represents the modal gain at threshold. In this way, a discontinuity would happen at the threshold point; therefore, unified analysis of $\mathrm{OB}$ in semiconductor lasers from below to above threshold could not be obtained. In our analysis, we directly solved the rate equations (1)-(3) numerically in the time domain using the fourth-order Runge-Kutta method. In order to make the result stable enough in the static properties' analysis, the calculated data in the time domain were averaged within $10 \mathrm{~ns}$ after $30 \mathrm{~ns}$ from turn-on. With a definite input optical power and by sweeping the input signal frequency detuning up and down, we can obtain optical bistability for the output optical power. Of course the $O B$ effect depends closely on the bias level of the laser. The calculated bistable loop width versus the normalized injection current of the slave laser, from below to above threshold, is given in Fig. 1 for three different values of injected optical power. The parameters used in obtaining Fig. 1 are $\alpha=6, G_{N}=5.04 \times 10^{3} \mathrm{~s}^{-1}, G_{I}=3.4 \times 10^{5} \mathrm{~s}^{-1}$, $N_{0}=10^{8}, \tau_{i}=5.5 \mathrm{ps}, \tau_{i}=1.2 \mathrm{ps}, A=10^{8} \mathrm{~s}^{-1}, B=$
$1 \mathrm{~s}^{-1}, C=10^{-9} \mathrm{~s}^{-1}, \beta_{\mathrm{sp}}=10^{-5}$, and $v=10^{-10} \mathrm{~cm}^{3}$. These are the typical values in the bulk distributedfeedback semiconductor lasers. The injected optical powers $P_{\text {in }}$ in Fig. 1 are $-25 \mathrm{dBm}$ (short-dashed curve), $-23 \mathrm{dBm}$ (solid curve), and $-21 \mathrm{dBm}$ (longdashed curve), where $P_{\text {in }}=\left|E_{\text {ext }}\right|^{2} h \nu$, with $h \nu$ the photon energy. The calculated results agree well with the experiment. It is also evident from Fig. 1 that the maximum point of $\mathrm{OB}$ versus injection current increases with the injected optical power, and the best $\mathrm{OB}$ results are obtained when the slave laser is polarized at a level a little above its free-running threshold. This is consistent with a previous prediction that optical input can only increase the lasing threshold. ${ }^{10}$ The calculation revealed that the spontaneous emission coefficient $\beta_{\mathrm{sp}}$ also plays an important role in determining the $\mathrm{OB}$ properties near threshold, and this is shown in Fig. 2. Spontaneous emission of the slave laser tends to damp the nonlinear effect in the threshold region.

In order to complete our analysis, we also investigated the optical switch characteristic of $O B$ in the time domain using the same parameters as in Fig. 1. The slave laser's injection current ranges from $I=$ $20 \mathrm{~mA}$ to $I=24 \mathrm{~mA}$, while the threshold current is $I_{\text {th }}=21.3 \mathrm{~mA}$. For the calculations to be comparable with the previous study ${ }^{10}$ and practical situations, the Gaussian input optical pulse is chosen with a FWHM of $2 \mathrm{~ns}$ and a peak power of $0.8 \mathrm{~mW}$ centered at $t=15 \mathrm{~ns}$. The initial frequency detuning of the master from the slave is kept the same. Figure 3(a) shows the optical output signal. In order to have a clear presentation, the curves are delayed from each other by $1 \mathrm{~ns}$. In addition to the previous observation that the switch-off time becomes smaller as the injection current is increased toward the threshold level, ${ }^{10}$ we found that the switch-off can be even faster when the slave laser is biased above threshold. Figure 3(b) illustrates the relative variation of the carrier population inside the slave laser's active

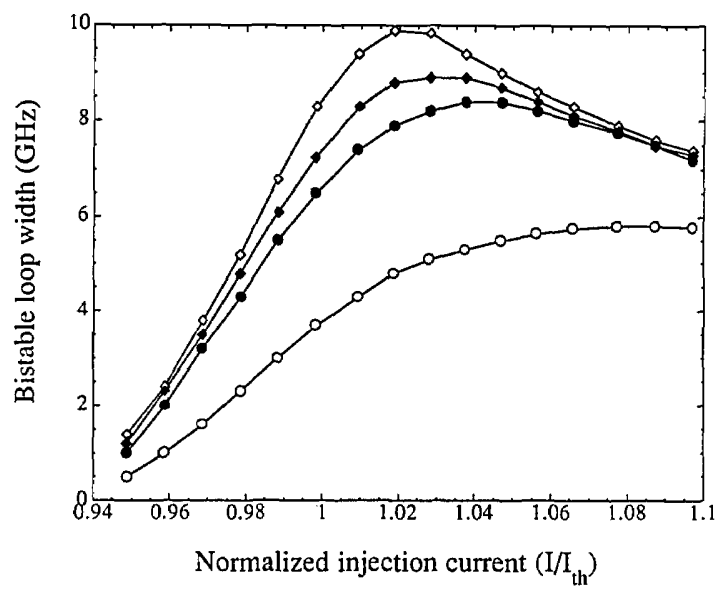

Fig. 2. Calculated bistable loop width versus the normalized injection current with the slave laser's spontaneous emission coefficient $\beta_{\mathrm{sp}}$ as the parameter: $\beta_{\mathrm{sp}}=3.5 \times 10^{-5}$ (open diamonds), $\beta_{\mathrm{sp}}=1.75 \times 10^{-4}$ (filled diamonds), $\beta_{\mathrm{sp}}=3.5 \times 10^{-4}$ (filled circles), $\beta_{\mathrm{sp}}=$ $1.75 \times 10^{-3}$ (open circles). $I_{\text {th }}$ is the free-runing laser threshold. 

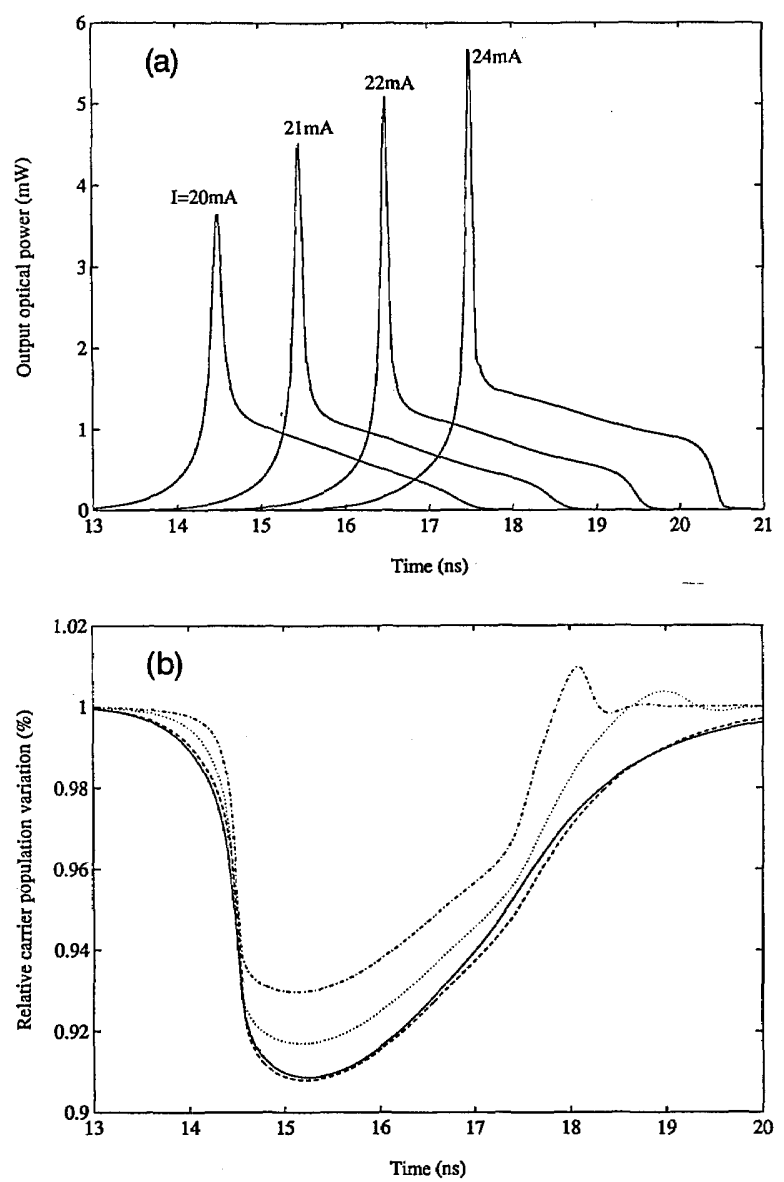

Fig. 3. (a) Calculated output pulse from $\mathrm{OB}$ element with increasing electric bias current from $I=20 \mathrm{~mA}$ to $I=24 \mathrm{~mA}$. The threshold current of the slave laser is $I_{\text {th }}=21.3 \mathrm{~mA}$. The curve has been delayed for $1 \mathrm{~ns}$ for $I=21 \mathrm{~mA}, 2 \mathrm{~ns}$ for $I=22 \mathrm{~mA}$, and $3 \mathrm{~ns}$ for $I=24 \mathrm{~mA}$ in order to have a better representation. (b) Calculated relative variation of the carrier population when the slave laser was biased at $I=20 \mathrm{~mA}$ (solid curve), $21 \mathrm{~mA}$ (dashed curve), $22 \mathrm{~mA}$ (dotted curve), and $24 \mathrm{~mA}$ (dashed-dotted curve).

cavity. It is evident that below threshold the carrier recovery at switch-off is determined mainly by the effective carrier lifetime ( $\sim 2 \mathrm{~ns})$. Above threshold, however, this carrier recovery can be much faster. A carrier recovery as fast as $0.5 \mathrm{~ns}$ has been obtained when the laser was biased at $24 \mathrm{~mA}$. The physical mechanism behind this fast switching is that at switch-off transient there is a competition between the amplified input optical signal and the amplified spontaneous emission of the slave laser. The carrier dynamics is now no longer governed by the effective carrier lifetime; rather, it is determined by the inverse of the relaxation oscillation frequency of the slave laser. Since the carrier recovery time is slower than the optical switch-off time, the time constant that limits the transition speed should be the former. Therefore, a much faster switch-off can be obtained if the slave laser is biased at relatively high levels. Another advantage of using the $\mathrm{OB}$ in the injection- locked condition is that, as we can see from Fig. 3(a), the output optical power is higher than in the case in which the slave laser operates below threshold and the $O B$ switch-off becomes clearer. However, the harder the slave laser is biased, the more optical signal is required to be injected into the slave laser to achieve the bistable operation, ${ }^{6}$ which thus sets a limitation on the slave laser's bias level. From the point of view of applications, since the slave laser operates above threshold, an optical filter is usually required to remove the stimulated emission from the slave laser itself. At switch-off, the frequency difference between the signal and the free-runing slave mode can be at the order of tens of gigahertz, depending on the ratio between the injected optical power and the power of the slave laser. ${ }^{6}$ A numerical calculation of the dynamic properties of diode-laser bistability above threshold has been recently given in Ref. 11, in which the gain saturation was neglected. We found, however, that the effect of gain saturation is essential in damping the relaxation-oscillationinduced instability at switching.

In conclusion, a unified analysis of dispersive $\mathrm{OB}$ in a semiconductor laser operating from below to above threshold has been performed. The result can be useful in obtaining a better understanding of the $O B$ performance in semiconductor lasers and in optimizing the condition for $\mathrm{OB}$ operation. The switch-off time of $\mathrm{OB}$ much less than the effective carrier lifetime can be obtained when the laser operates in the injection-locked regime.

This research was supported by the Consiglio Nazionale delle Ricerche and Camera di Commercio di Torino under research grant All-Optical Communication Networks. The authors thank N. Caponio, P. Gambini, M. Puleo, and E. Vezzoni for helpful discussions.

\section{References}

1. K. Otsuka and H. Iwamura, IEEE J. Quantum Electron. QE-19, 1184 (1983).

2. K. Otsuka and S. Kobayashi, Electron. Lett. 19, 262 (1983).

3. A. J. Adams, H. J. Westlake, M. J. Mahony, and I. D. Henning, IEEE J. Quantum Electron. QE-21, 1498 (1985).

4. M. J. Adams, IEE Proc. Pt. J 132, 343 (1985).

5. H. Kawaguchi, K. Inoue, T. Matsuoka, and K. Otsuka, IEEE J. Quantum Electron. QE-21, 1314 (1985).

6. R. Hui, A. D'Ottavi, A. Mecozzi, and P. Spano, IEEE J. Quantum Electron. 27, 1688 (1991).

7. R. Lang, IEEE J. Quantum Electron. QE-18, 976 (1982).

8. R. Hui, A. Mecozzi, A. D'Ottavi, and P. Spano, Electron. Lett. 26, 997 (1990).

9. I. Petitbon, P. Gallion G. Debarge, and C. Chabran, IEEE J. Quantum Electron. 24, 148 (1988).

10. W. F. Sharfin and M. Dagenais, IEEE J. Quantum Electron. QE-23, 303 (1987).

11. M. Schell and E. Scholl, IEEE J. Quantum Electron. 26, 1095 (1990). 